\title{
Aktuelle Informationen zur Vertragsgestaltung Hämophilie und seltene Faktorenmängel
}

Da aktuell zahlreiche Entwicklungen mit sehr großer Dynamik seit Anfang März 2019 bzgl. der sogenannten „Behandlungsverträge“ eingesetzt haben, die auch der Vorstand des BDDH aktiv begleitet, möchte der Vorstand des BDDH informieren.

VDEK-Vertrag, aktualisierte Anlage E (Wirtschaftlichkeit der Faktorpräparate innerhalb der Verträge nach § 47 Abs. 1 Nr. 2a AMG):

Alle Preisgestaltungen wurden nun einheitlich in Rabattverträge nach $\S 130$ a Abs. 8 SGB V oder nach $\S 130$ c SGB V überführt. Damit ist eine rechtlich eindeutige und wirksame Grundlage etabliert.

Alle relevanten Faktorenpräparate für die Therapie der Hämophilie A und B sind als besonders wirtschaftlich eingestuft worden (grün). Die drei im AMNOG-Verfahren befindlichen Präparate werden möglicherweise noch folgen (Refixia ${ }^{\circledR}$, Adynovi ${ }^{\circledR}$, Jivi ${ }^{\circledR}$ ), wenn die Preisrunden abgeschlossen sind. Gleiches gilt ggf. auch für Hemlimbra ${ }^{\circledR}$ entsprechend der neu zugelassenen Indikation.

Die „auslaufenden“ Gerinnungsfaktorenpräparate (Berinin ${ }^{\circledR}$, Helixate ${ }^{\circledR}$, Kogenate $\left.{ }^{\circledR}\right)$ werden nicht mehr verhandelt, werden abverkauft und sind als nicht mehr besonders wirtschaftlich eingestuft. Die drei Präparate, (Wilate ${ }^{\circ}$, Haemate ${ }^{\circ}$, Voncento $\left.{ }^{\circ}\right)$ die sowohl für die Hämophilie A-Therapie und die Therapie des von-Willebrand-Syndrom zugelassen sind, sind sinnvollerweise für die Indikation der Hämophilie A als weniger wirtschaftlich in dieser Indikation gelistet. Bei der Indikation der Therapie des von-WillebrandSyndroms sind alle zugelassenen Gerinnungsfaktorenpräparate frei einsetzbar. Zwei pharmazeutische Unternehmen haben sogar freiwillig für zwei Gerinnungsfaktorenpräparate zur Therapie des von-Willebrand-Syndroms (vWS) einen weiteren Rabattvertrag geschlossen.

Die Laufzeit beträgt mindestens sechs Quartale, bis gegebenfalls ein neuer Vertriebsweg für Gerinnungsfaktorenkonzentrate nach dem Gesetz für mehr Sicherheit in der Arzneimittelversorgung (GSAV) vorgeschrieben wird. Und für die Zeit ab 01.07.2020 gibt es schon eindeutige, kongruente Vorstellungen zwischen dem VDEK und dem BDDH sowie der GTH als konzertierte Aktion für ein aktualisiertes Vertragsformat.

Fazit:

Dies bedeutet ein exzellente Basis für die behandelnden Zentren. Es muss kein Patient „umgestellt“ werden bei vollem Erhalt der Wirtschaftlichkeitspauschale und juristisch eindeutig geregelten Verträgen zwischen den Kassen und Zentren sowie zwischen den Kassen und den pharmazeutischen Unternehmen.

Die weiteren Krankenkassen ohne vertragliche Bindung (alle Betriebskrankenkassen und weitere Krankenkassen) werden aktuell durch die ServicePlus AG Gesellschaft für Wirtschaftlichkeit und Qualität bei Krankenkassen (GWQ), stellvertretend für 40 Krankenkassen, und SpectrumK GmbH, stellvertretend für 60 Krankenkassen, vertreten.

Es fanden im März 2019 die ersten Treffen mit beiden Beratungsgesellschaften statt und folgende weitreichenden Ergebnisse wurden vereinbart:

GWQ und SpectrumK werden den aktuellen, sogenannten VDEK-Vertrag als Vertragsgrundlage übernehmen. Die GWQ will ab 01.07.2019 mit den Verträgen starten. SpectrumK will starten, sobald alle von ihnen betreuten Krankenkassen eingebunden sind. Des Weiteren werden GWQ und SpectrumK national einheitliche Rabattverträge für die je ca. 8,5 Mio. und ca. 9,5 Mio. Versicherten ihrer jeweils betreuten Krankenkassen mit ALLEN pharmazeutischen Unternehmen aushandeln.

Also auch hier werden voraussichtlich die nächsten sechs Quartale vertraglich geregelt sein.

Die Zeit ab 01.07.2020 soll auch dem Vorbild des gegebenenfalls aktualisierten VDEK-Vertrages in Absprache mit dem BDDH und der GTH geregelt werden.

Fazit:

Alle Krankenkassen außerhalb des AOK-Bereiches haben und werden ein weitestgehend gleiches Vertragswerk anwenden und werden bereits erheblich von Rabattverträgen mit der pharmazeutischen Industrie schon vor dem 01.07.2020 profitieren.

Aktuelle Informationen zum Stand des Vertriebsweges für Gerinnungsfaktorenkonzentrate nach dem Gesetz für mehr Sicherheit in der Arzneimittelversorgung (GSAV) liegen nun auch vor.

Am 27.02.2019 wurde das GSAV intern im Gesundheitsausschuss des Bundesrates beraten. Im Antrag 2 wurden die geplanten Änderungen der Hämophilieversorgung im GSAV kritisiert. „Der Bundesrat befürchtet, dass die mit dem Gesetzentwurf geplanten Änderungen eine Schwächung der Zentrumsversorgung zur Folge hätte, weil die enge Bindung zwischen Zentrum und Patient durchbrochen würde.“

Der Bundesrat bittet daher die Bundesregierung die geplanten Neuregelung im weiteren Gesetzgebungsverfahren zu überprüfen. Der Antrag wurde knapp angenommen (8 Zustimmungen, 5 Ablehnungen, 3 Enthaltungen) und wird damit in die Stellungnahme des Bundesrates zum GSAV mit einfliessen, die dann in der Sitzung am 15. März beschlossen und an die Bundesregierung überwiesen wurde. Diese wird

(C) 2019 Georg Thieme Verlag KG Stuttgart . New York
DOI https://doi.org/

10.1055/s-0039-1688544. ISSN 0720-9355. 
dann eine Gegendarstellung formulieren und sich zu den Forderungen des Bundesrates positionieren.

Die Gegenäußerung der Bundesregierung zur Stellungnahme des Bundesrates zum GSAV kam Ende März 2019. Demnach wird die Bundesregierung an den vorgesehen Änderungen des Vertriebsweges festhalten:

"Die Bundesregierung hält an der vorgesehenen Änderung des Vertriebswegs für Arzneimittel zur Behandlung von Gerinnungsstörungen bei Hämophilie fest. Die Änderung des Vertriebsweges für Gerinnungsfaktorenzubereitungen wurde erforderlich durch die Entwicklung bzw. Zulassung neuer Arzneimittelgruppen (insbesondere monoklonaler Antikkörper), die nicht der bisherigen Regelung zum Direktvertrieb für Gerinnungsfaktorenzubereitungen zugeordnet werden konnten. Zur Vermeidung von Marktverzerrungen werden alle Arzneimittel zur spezifischen Therapie von Gerinnungsstörungen bei Hämophilie dem regulären Apothekenvertrieb zugeordnet. Gleichzeitig wird das Dispensierrecht der Ärzte in diesem Bereich aufgehoben, mit Ausnahme der Bevorratung und Abgabe zur Notfallversorgung.

Die Versorgungsstruktur durch Hämophiliezentren sowie durch spezialisierte Vertragspraxen ist durch die Umstellung der Vertriebsstruktur der Arzneimittel nicht gefährdet, da die Krankenkassen oder ihre Landesverbände verpflichtet werden, mit Hämophiliezentren oder auch spezialisierten Vertrag- spraxen Verträge über die Behandlung von Versicherten mit Gerinnungsstörungen bei Hämophilie zu schließen. In diesen Verträgen sollen insbesondere die Vergütung von zusätzlichen ärztlichen Aufwendungen vereinbart werden."

Der Gesetzesentwurf geht nun in den Gesundheitsausschuss des Bundestags. Dort findet die öffentliche Anhörung am 10. April von 14 bis $16 \mathrm{Uhr}$ statt. Voraussichtlich wird es spezifische Fragen von Abgeordneten zum Hämophiliethema geben.

Da dieser Teil des GSAV vom Bundesrat zustimmungspflichtig ist, wird die endgültige, zukünftige Abstimmung im Bundesrat entscheidend sein.

Autor für den BDDH:

Priv.-Doz. Dr. med. Jürgen Koscielny

Leiter der Gerinnungsambulanz mit Hämophiliezentrum im ambulanten Gesundheitszentrum (AGZ) der Charité $\mathrm{GmbH}$

Charité - Universitätsmedizin Berlin, Campus Charité Mitte (CCM)

Charitéplatz 1, Durchgang Luisenstr. 13

D-10117 Berlin (Germany)

e-mail: juergen.koscielny@charite.de 